ARTICLE

DOI: $10.1038 / s 41467-018-04988-x$

\title{
Evidence for line width and carrier screening effects on excitonic valley relaxation in 2D semiconductors
}

\author{
Yuhei Miyauchi 10 1,2, Satoru Konabe 3,10, Feijiu Wang ${ }^{1,2}$, Wenjin Zhang ${ }^{1}$, Alexander Hwang ${ }^{4,5}$, \\ Yusuke Hasegawa1, Lizhong Zhou', Shinichiro Mouri ${ }^{1,6}$, Minglin Toh7, Goki Eda (D) ${ }^{7,8,9}$ \& Kazunari Matsuda
}

Monolayers of transition metal dichalcogenides (TMDC) have recently emerged as excellent platforms for exploiting new physics and applications relying on electronic valley degrees of freedom in two-dimensional (2D) systems. Here, we demonstrate that Coulomb screening by $2 \mathrm{D}$ carriers plays a critical role in excitonic valley pseudospin relaxation processes in naturally carrier-doped $\mathrm{WSe}_{2}$ monolayers $\left(1 \mathrm{~L}-\mathrm{WSe}_{2}\right)$. The exciton valley relaxation times were examined using polarization- and time-resolved photoluminescence spectroscopy at temperatures ranging from 10 to $160 \mathrm{~K}$. We show that the temperature-dependent exciton valley relaxation times in $1 \mathrm{~L}-\mathrm{WS} \mathrm{S}_{2}$ under various exciton and carrier densities can be understood using a unified framework of intervalley exciton scattering via momentum-dependent longrange electron-hole exchange interactions screened by 2D carriers that depend on the carrier density and the exciton linewidth. Moreover, the developed framework was successfully applied to engineer the valley polarization of excitons in $1 \mathrm{~L}-\mathrm{WSe} \mathrm{S}_{2}$. These findings may facilitate the development of TMDC-based opto-valleytronic devices.

\footnotetext{
${ }^{1}$ Institute of Advanced Energy, Kyoto University, Uji, Kyoto 611-0011, Japan. ${ }^{2}$ Graduate School of Science, Nagoya University, Chikusa, Nagoya 464-8602, Japan. ${ }^{3}$ Research Institute for Science and Technology, Tokyo University of Science, 6-3-1 Katsushika-ku, Tokyo 125-8585, Japan. ${ }^{4}$ Department of Electrical and Computer Engineering, Rice University, Houston, TX 77005, USA. ${ }^{5}$ Department of Physics, Rice University, Houston, TX 77005, USA. ${ }^{6}$ Department of Electrical and Electronic Engineering, Ritsumeikan University, Kusatsu 525-8577, Japan. ${ }^{7}$ Department of Physics, National University of Singapore, Singapore 117551, Singapore. ${ }^{8}$ Centre for Advanced 2D Materials and Graphene Research Centre, National University of Singapore, Singapore 117546, Singapore.

${ }^{9}$ Department of Chemistry, National University of Singapore, Singapore 117543, Singapore. ${ }^{10}$ Present address: Department of Chemical Science and Technology, Hosei University, Koganei, Tokyo 184-8584, Japan. Correspondence and requests for materials should be addressed to

Y.M. (email: miyauchi@iae.kyoto-u.ac.jp) or to S.K. (email: konabe@hosei.ac.jp)
} 
A

"valley" is an electronic degree of freedom in momentum space, and its potential applications as information carriers in future electronics or optoelectronics devices are called valleytronics ${ }^{1-4}$. Monolayers of transition metal dichalcogenides (1L-TMDCs) $M X_{2}(M=\mathrm{Mo}, \mathrm{W} ; X=\mathrm{S}$, Se, etc.) have recently emerged as promising two-dimensional (2D) materials for developing valleytronics because they have hexagonal lattice structures similar to that of graphene but are semiconductors with finite direct band gaps in two inequivalent $+K$ and $-K$ valleys related by a time-reversal operation in the $2 \mathrm{D}$ hexagonal Brillouin zone $e^{5,6}$. Because of the reduced screening resulting from the atomically thin $2 \mathrm{D}$ structures of $1 \mathrm{~L}-\mathrm{TMDCs}$, their excitons, which are mutually attracting electron-hole pairs that interact through Coulomb interactions, have extremely large binding energies and dominate their optical responses even at room temperature ${ }^{7-10}$. In addition, the strong spin-orbit interactions in these materials give rise to large spin splitting, reaching $\sim 450$ $\mathrm{meV}(\sim 150 \mathrm{meV})$ for $\mathrm{W} X_{2}\left(\mathrm{MoX}_{2}\right)$ in the valence band ${ }^{11}$. This large valence spin splitting and a lack of inversion symmetry in these materials lead to spin-valley coupling that enables exclusive access to the excitonic valley pseudospins $(|+K\rangle$ or $|-K\rangle)$ with right- or left-circularly polarized photons ${ }^{12-16}$. These unique characteristics of 1L-TMDCs have provided unprecedented platforms for the study of valley-exciton physics in $2 \mathrm{D}$ systems, as well as offering opportunities for developing future optoelectronic devices using the excitonic valley degrees of freedom $^{4,12-15,17-33}$.

One of the most intriguing questions in valley-exciton physics is in regard to the relaxation mechanism of excitonic valley pseudospins in 1L-TMDCs ${ }^{4,12-15,17-23,28,30-32,34-48}$. With regard to the theory on the subject, exciton (or hole) valley relaxations dominated by electron-hole $(e-h)$ exchange interactions ${ }^{14,19,20,32,38,39}$, as well as phonon-assisted intervalley scattering mechanisms ${ }^{13,17,37}$, have been proposed and intensively studied. The importance of the Coulomb screening effect by naturally or intentionally doped carriers in 1L-TMDCs has also been examined theoretically ${ }^{32}$, although it has not yet been addressed experimentally. With regard to investigating the question experimentally, time- and polarization-resolved transient absorption ${ }^{19,22}$, reflection ${ }^{35}$, photoluminescence $(\mathrm{PL})^{28,41}$, and Faraday ${ }^{43}$ and Kerr rotation (TRKR) ${ }^{38,44,46,47,49-51}$ techniques have been used to measure the depolarization (life) times of the spin or exciton (hole) valley states in $1 \mathrm{~L}-\mathrm{WSe}_{2}{ }^{38,46,47,50,52}, 1 \mathrm{~L}-$ $\mathrm{WS}_{2}{ }^{22,49}$, and $1 \mathrm{~L}-\mathrm{MoS}_{2}{ }^{19,35,43,44,51}$, and spin or valley relaxation times ranging from several to $10 \mathrm{ps}^{38,46,47}$ to tens of nanoseconds ${ }^{50}$ have been reported at cryogenic temperatures. The considerable variations in the reported spin or valley relaxation times have been discussed as being possible consequences of either the differences between the physical quantities observed by each measurement technique ${ }^{49}$, the existence of dark exciton states ${ }^{49}$, the spin/valley polarization of resident holes ${ }^{50,52}$, ultrafast Coulomb-induced intervalley coupling ${ }^{45}$, the slow decay of localized states ${ }^{47}$, or the high excitation density typically needed for pump-probe-type experiments using femtosecond lasers (typically $>\sim 10^{12} \mathrm{~cm}^{-2}$ within the initial $100-200 \mathrm{fs}$ ) $28,46,49$. Because of all or some of these complications, a comprehensive and unifying explanation for temperature-dependent exciton
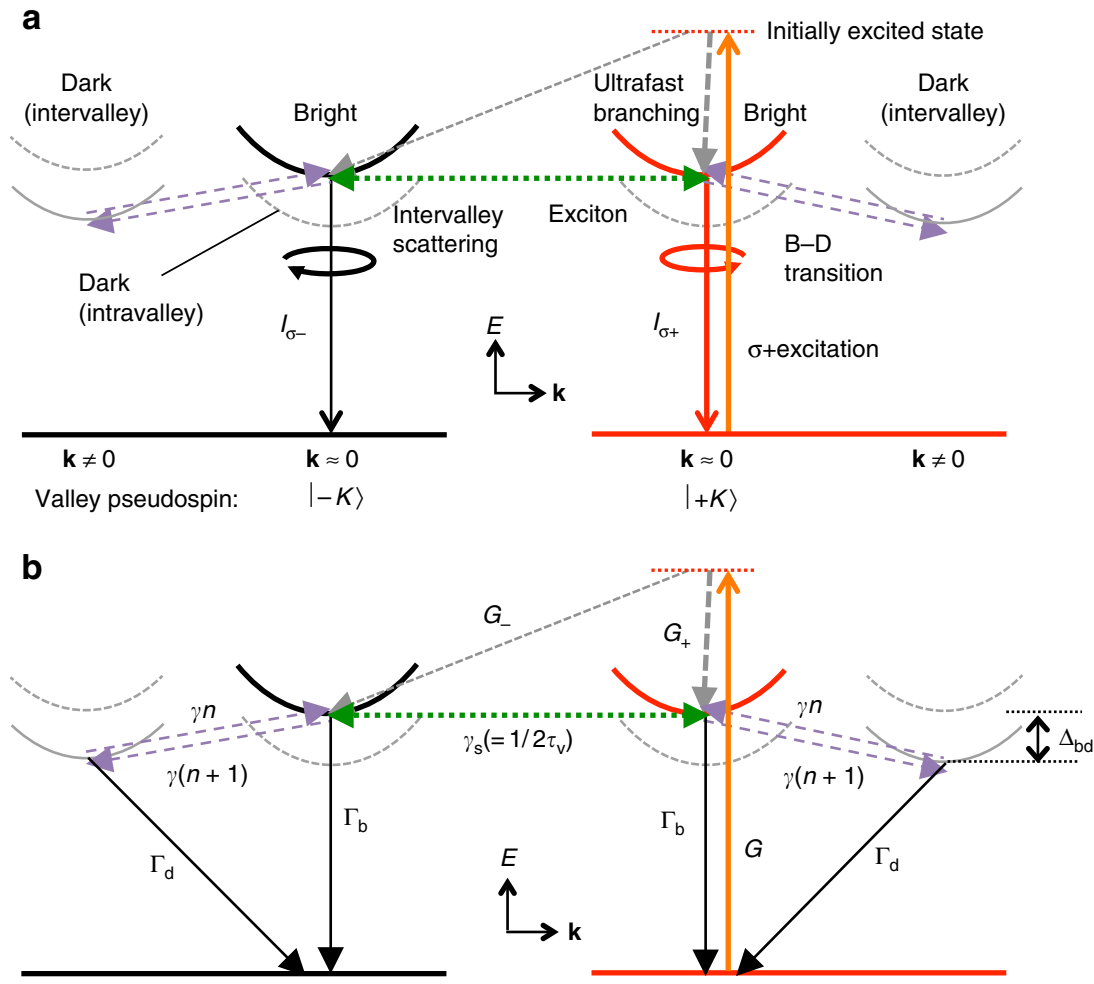

Fig. 1 Excitation and relaxation processes of valley-polarized excitons. a Schematic of the processes in the $|+K\rangle$ valley-pseudospin-selective excitation followed by circularly polarized PL from the bright $(\mathbf{k} \approx \mathbf{0})$ excitons with $|+K\rangle$ (red) or $|-K\rangle$ (black) valley pseudospins. Solid and dotted bands correspond to the singlet-like and triplet-like exciton bands, respectively. The horizontal axis corresponds to the exciton wave vector $\mathbf{k}$. Exciton bands at $\mathbf{k} \approx \mathbf{0}$ are at the zone center in the excitonic Brillouin zone. Intervalley dark excitons have $\mathbf{k} \neq \mathbf{0}$. Arrows indicate the major transition and scattering pathways assumed to be involved for the phenomena considered in this study. $\mathbf{b}$ Symbols denoting the rates for the exciton recombination and scattering processes considered in a. Parameter $\gamma$ is a phonon scattering rate constant, and $n$ is a phonon number available for the bright-dark (B-D) transitions with the energy difference of $\Delta_{b d}$. In the schematics of $\mathbf{a}$ and $\mathbf{b}$, only the intervalley B-D scattering pathways are indicated for clarity. However, we do not exclude the possibility of the contribution of the intravalley B-D scattering (see Supplementary Note 1 for additional details) 
a

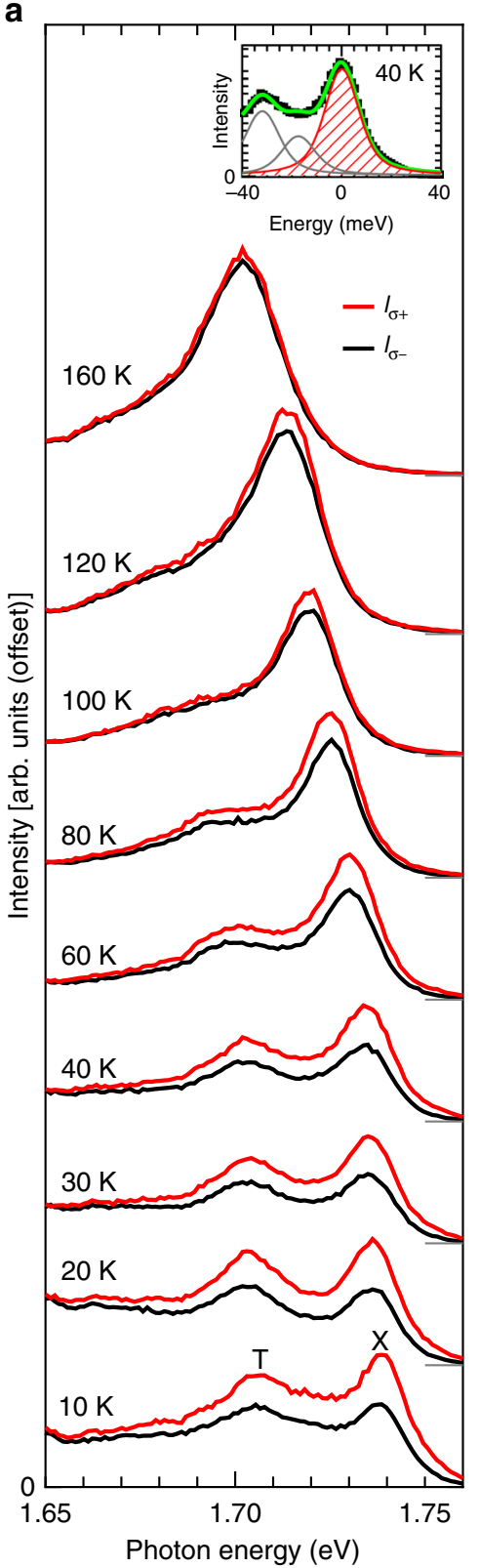

b

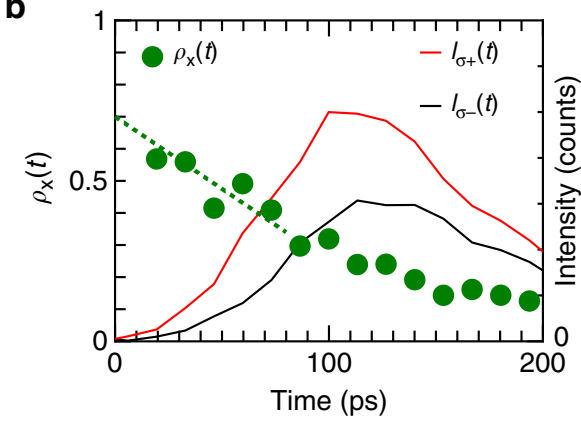

c

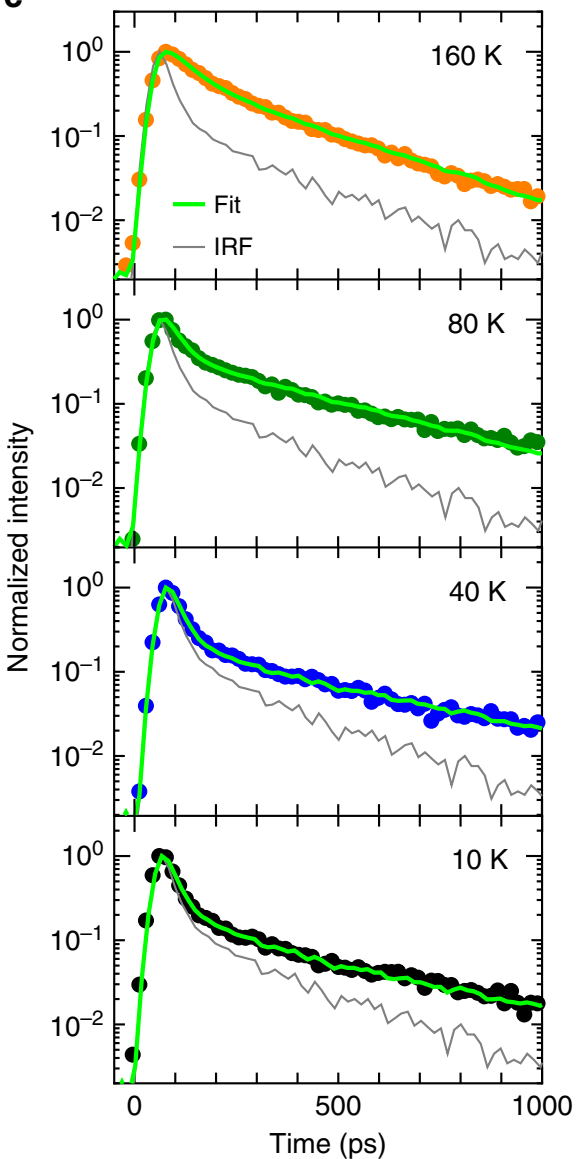

Fig. 2 Polarization- and time-resolved PL measurements at various temperatures. a Polarization-resolved PL spectra of $1 \mathrm{~L}-\mathrm{WS} \mathrm{e}_{2}$ on a quartz substrate under $\sigma_{+}$excitation at $10-160 \mathrm{~K}$. The red and black curves correspond to the $\sigma_{+}$and $\sigma_{-} \mathrm{PL}$ intensities, $I_{\sigma+}$ and $I_{\sigma-}$, respectively. The inset shows an example of the spectral fitting for the $I_{\sigma+}$ spectrum at $40 \mathrm{~K}$ using Voigt functions. The shaded peak corresponds to the exciton PL. $\mathbf{b} \rho_{x}$ as a function of time (solid circles) calculated from time $(t)$-resolved PL signals of $I_{\sigma+}(t)$ and $I_{\sigma-}(t)$ at $45 \mathrm{~K}$ measured for $1 \mathrm{~L}-\mathrm{WS} \mathrm{e}_{2}$ on a quartz substrate. c Time-resolved PL decay profiles of excitons measured at $160 \mathrm{~K}$ (orange), $80 \mathrm{~K}$ (green), $40 \mathrm{~K}$ (blue), and $10 \mathrm{~K}$ (black). Emission polarization was not resolved for these measurements. Light-green curves are fit to the data, and gray curves are the instrumental response function (IRF)

valley relaxation times that is applicable for various exciton and carrier density conditions has yet to be provided.

In the present paper, we provide experimental evidence that the excitonic valley relaxation times and their temperature dependence in naturally carrier-doped $1 \mathrm{~L}-\mathrm{TMDCs}$ are dominated by momentum-dependent $e-h$ exchange interactions screened by a $2 \mathrm{D}$ electron gas. We measured the valley polarization, linewidth, and time-dependent PL decay profile of excitons in $1 \mathrm{~L}-\mathrm{WSe}_{2}$ to examine the valley relaxation times of an exciton at temperatures ranging from 10 to $160 \mathrm{~K}$ in the linear response regime. We show that the low-temperature exciton valley relaxation times in 1L$\mathrm{WSe}_{2}$ can be excellently reproduced using a framework of an intervalley exciton scattering mechanism via momentumdependent long-range $e-h$ exchange interactions screened by naturally doped 2D carriers. The temperature dependence of the Coulomb screening function deduced from the experimental data clearly demonstrates the $2 \mathrm{D}$ nature of the doped carriers; this is a unique manifestation of the true $2 \mathrm{D}$ structure of $1 \mathrm{~L}$-TMDCs. Moreover, we demonstrate that the developed framework can be used to predict the valley relaxation times and polarizations under various experimental conditions with various exciton linewidths, exciton lifetimes, carrier densities, and exciton densities. We also demonstrate that the valley polarization in $1 \mathrm{~L}-\mathrm{WSe}_{2}$ can be actually engineered via artificially modifying these parameters 
a
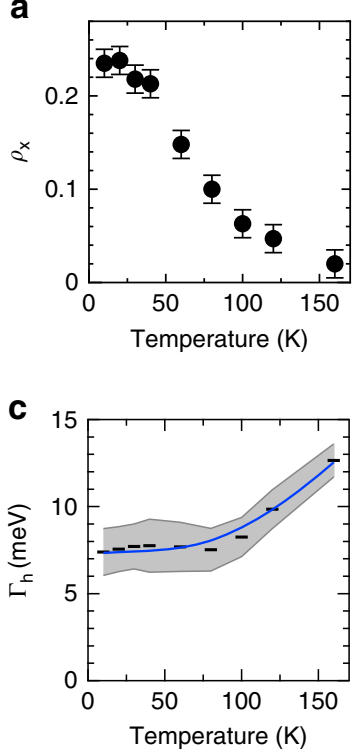

b

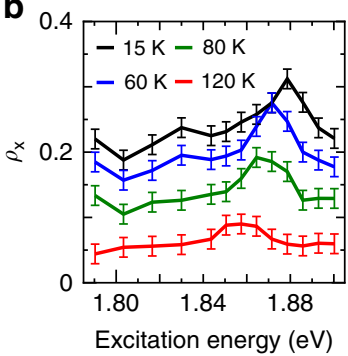

d

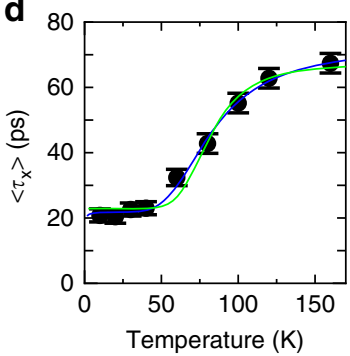

e

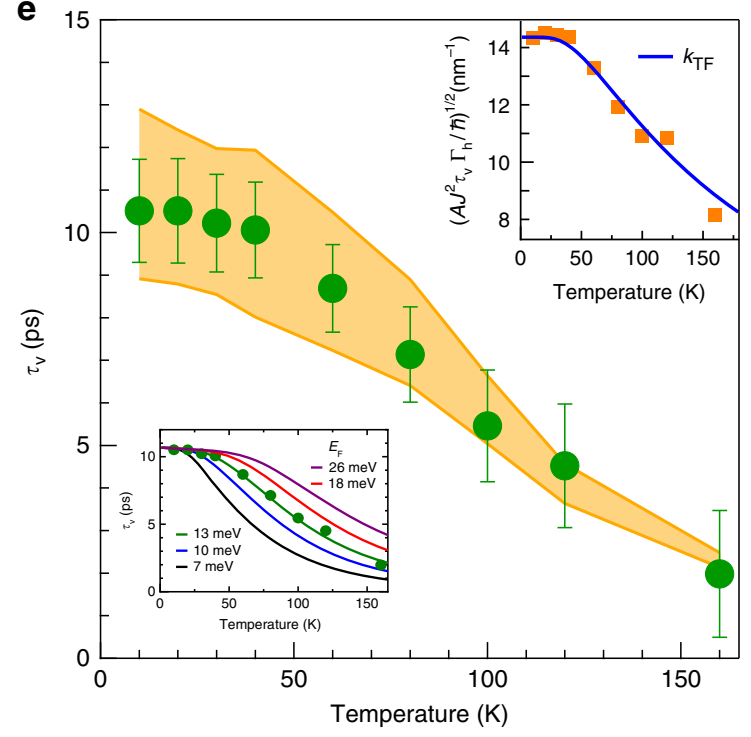

Fig. 3 Temperature dependence of exciton valley relaxation times. a Valley polarization $\rho_{\mathrm{x}}$ as a function of temperature. $\mathbf{b} \rho_{\mathrm{x}}$ at various temperatures as functions of excitation photon energy. The error bars in $\mathbf{a}, \mathbf{b}$ correspond to the uncertainties of the polarization-resolved measurements. $\mathbf{c} \Gamma_{\mathrm{h}}$ as a function of temperature. The gray-shaded region indicates the reasonable range of $\Gamma_{\mathrm{h}}$ deduced from the Voigt fitting procedure. Bars are the mean values in the reasonable range at each temperature. The blue curve is the empirical function obtained by the fit to the mean values. $\mathbf{d}\left\langle\tau_{x}\right\rangle$ as a function of temperature. Solid curves are the fitting results obtained using Eq. (5) with $\Delta_{\mathrm{bd}}$ of $30 \mathrm{meV}$ (blue curve) and $47 \mathrm{meV}$ (light-green curve). The error bars represent the uncertainties in the fitting procedure. e $\tau_{\vee}$ calculated using Eq. (1) and plotted as a function of temperature (solid circles). The error bars were estimated from the uncertainties in $\left\langle\tau_{\mathrm{x}}\right\rangle$ and $\rho_{\mathrm{x}}$ used for calculating $\tau_{\mathrm{v}}$. The orange-shaded region is the prediction band from Eq. (2) corresponding to the reasonable range of $\Gamma_{\mathrm{h}}$ in $\mathbf{c}$ with the fitting parameters of $J=0.83 \mathrm{eV}$ and $E_{\mathrm{F}}=13 \mathrm{meV}\left(n_{\mathrm{c}} \approx 2.1 \times 10^{12} \mathrm{~cm}^{-2}\right)$. The upper-right inset is a plot of $\left[A J^{2} \tau_{\mathrm{v}}(T) \Gamma_{\mathrm{h}}(T) / \hbar\right]^{1 / 2}$ as a function of temperature. The blue curve is the fitting result obtained by $k_{T F}\left(T, E_{\mathrm{F}}\right)$ with $E_{\mathrm{F}}=13 \mathrm{meV}$. The lower-left inset shows the predicted temperature dependence of $\tau_{\mathrm{v}}$ for various $E_{\mathrm{F}}$ (carrier density), as obtained using Eq. (3). For the $\Gamma_{\mathrm{h}}$, the empirical fit function plotted in c (blue curve) was used for clarity

according to the theoretical prediction. Our findings therefore provide a unified framework through which the temperaturedependent exciton valley relaxation times and valley polarization in 1L-TMDCs can be understood and predicted; this framework may facilitate the development of TMDC-based opto-valleytronic devices.

\section{Results}

Measurements of exciton valley polarization. Figure 1a shows a schematic of the excitation of optically allowed (bright) excitons with the center-of-mass wave vector $\mathbf{k} \approx \mathbf{0}$ in the excitonic Brillouin zone and the valley pseudospin $|+K\rangle$ (for which both electron and hole are in the $+K$ valley in the electronic Brillouin zone), initial ultrafast decays partially branching into bright excitons with the valley pseudospin $|+K\rangle$ or $|-K\rangle$ (for which both electron and hole are in the $-K$ valley in the electronic Brillouin zone), the intervalley scattering between the $|+K\rangle$ and $|-K\rangle$ states causing exciton valley relaxation, circularly polarized emission processes from the $|+K\rangle$ and $|-K\rangle$ bright excitons, and the electron spin-conserving scattering between the bright $(\mathbf{k} \approx \mathbf{0})$ and dark $(\mathbf{k} \neq \mathbf{0})$ exciton states in $1 \mathrm{~L}-\mathrm{WSe}_{2}$. The dark excitons with $\mathbf{k} \neq \mathbf{0}$ indicated by gray solid curves in Fig. 1 are optically forbidden because of the momentum mismatch with photons. Symbols $\sigma_{+}$and $\sigma_{-}$denote the circular polarizations, and $I_{\sigma+}$ and $I_{\sigma-}$ represent the corresponding PL intensities. Figure $1 \mathrm{~b}$ shows the scattering processes and symbols for the relevant rates considered in this study. The valley relaxation time $\left(\tau_{\mathrm{v}}\right)$ of the bright exciton is related to its intervalley scattering rate $\left(\gamma_{\mathrm{s}}\right)$ as $\tau_{\mathrm{v}}=\left(2 \gamma_{\mathrm{s}}\right)^{-1}$. $G\left(=G_{+}+G_{-}\right)$is the generation rate of the bright excitons. Parameters $G_{+}$and $G_{-}$are the effective generation rate of the bright excitons with $|+K\rangle$ and $|-K\rangle$ pseudospins after the initial branching within the ultrafast timescale, respectively. We define $\rho_{0} \equiv\left(G_{+}-G_{-}\right) /\left(G_{+}+G_{-}\right)$to express the valley polarization loss in the initial ultrafast non-thermal timescale. In the ideal case under $\sigma_{+}$excitation, such as the resonant excitation condition to the bright exciton state, $\rho_{0}$ should be equal to 1 because of the optical selection rule ${ }^{12-15}$. However, $\rho_{0}$ could be less than 1 in the event of an initial depolarization process due to defect-induced intervalley generation ${ }^{35}$, ultrafast valley relaxation of hot excitons during their intraband cooling processes ${ }^{39}$, and/or excitation to other spin-degenerate valleys ${ }^{53}$, potentially through phononassisted indirect absorption processes ${ }^{37}$. In Fig. 1, the exciton scattering processes between the bright and intervalley dark states by phonon-assisted spin-conserving electron scattering processes $^{54}$ are tentatively indicated as a major bright-dark transition pathway. In this study, however, we do not attempt to distinguish the microscopic origin of the lower-lying dark states (intervalley spin-allowed or intravalley spin-forbidden) for the analysis under the condition that the hole's valley is conserved. Recent experimental studies have revealed that the energy difference between the bright and dark states $\left(\Delta_{\mathrm{bd}}\right)$ in $1 \mathrm{~L}-\mathrm{WSe}_{2}$ is $\sim 30-47 \mathrm{meV}^{54-57}$. For the valley depolarization of holes (excitons), we consider the scattering between the singlet-like bright $(\mathbf{k} \approx \mathbf{0})$ excitons with $|+K\rangle$ or $|-K\rangle$ valley pseudospins via longrange $e-h$ exchange interactions ${ }^{32,39}$ to be the dominant valley depolarization mechanism. Because this mechanism is effective only between the two bright states with opposite valley pseudospins, the hole valley relaxation of dark excitons is neglected as a first approximation in this study (see Supplementary Note 1 for additional details).

Figure 2a shows the polarization-dependent PL spectra of 1L$\mathrm{WSe}_{2}$ on a quartz substrate under circularly polarized $\sigma_{+}$ excitation conditions at $10-160 \mathrm{~K}$. At low temperatures, an exciton (A-exciton) peak $(\mathrm{X} \text {, at } 1.740 \mathrm{eV})^{34,58}$ and a charged exciton (trion) peak $(\mathrm{T} \text {, at } 1.705 \mathrm{eV})^{34}$ were observed. The emergence of the trion peaks and their peak positions indicate 
that $1 \mathrm{~L}-\mathrm{WSe}_{2}$ was in a naturally electron-doped condition ${ }^{34,59}$. The difference between the spectral integrated intensities of $\sigma_{+}$ $\left(I_{\sigma+}\right)$ and $\sigma_{-}\left(I_{\sigma-}\right)$ exciton PL (valley polarization) was clearly observed at low temperatures. Under the excitation by $\sigma_{+}$photons, the valley polarization, $\rho_{\mathrm{x}}$, is calculated as $\rho_{\mathrm{x}}=\left(I_{\sigma+}-I_{\sigma}-\right) /\left(I_{\sigma+}+\right.$ $\left.I_{\sigma-}\right)$. As shown in Fig. $3 \mathrm{a}, \rho_{\mathrm{x}}$ values were nearly constant under the low-temperature conditions $(T<\sim 40 \mathrm{~K})$ and gradually decreased with increasing temperature for $T>\sim 60 \mathrm{~K}$. Similar temperaturedependent behaviors of $\rho_{\mathrm{x}}$ have been reported for $1 \mathrm{~L}-\mathrm{MoS}_{2}{ }^{13}$ and $1 \mathrm{~L}-W \mathrm{We}_{2}$ (for $\left.T \geq 70 \mathrm{~K}\right)^{31}$.

The excitation photon energy dependences of $\rho_{\mathrm{x}}$ at various temperatures were also examined; the results are shown in Fig. $3 \mathrm{~b}$. Relatively high values of $\rho_{\mathrm{x}}$ were observed at the near-resonant excitation conditions with the $2 s$ excited exciton state ${ }^{8,9}$ lying at an energy $\sim 0.14 \mathrm{eV}$ higher than the $1 s$ excitons at $1.74 \mathrm{eV}$, whereas variations in $\rho_{\mathrm{x}}$ were small under the nonresonant conditions (corresponding to excitation photon energies of $\sim 1.79-1.84 \mathrm{eV}$ and $1.89-1.90 \mathrm{eV}$ ) within the measured range from 1.79 to $1.90 \mathrm{eV}$. Because we employed nonresonant excitation conditions (the excitation photon energy was 1.893 $\mathrm{eV}$ ) for evaluating the temperature dependence of $\rho_{\mathrm{x}}$ shown in Fig. 3a, we ruled out the possibility that the observed temperature dependence in $\rho_{\mathrm{x}}$ was determined by the temperature-dependent exciton energy shift ${ }^{60}$, which had previously been discussed as being the case in $1 \mathrm{~L}-\mathrm{MoS}_{2}{ }^{14,40}$. The excitation photon energy dependence of $\rho_{\mathrm{x}}$ at low temperature is discussed in greater detail in Supplementary Note 2 and Supplementary Figure 1.

To deduce the valley polarization loss in the initial ultrafast non-thermal timescale (the value of $\rho_{0}$ ) for the following analysis, we also measured polarization-resolved PL decay profiles of the excitons in $1 \mathrm{~L}-\mathrm{WSe}_{2}$ on a quartz substrate. Figure $2 \mathrm{~b}$ shows the polarization-resolved PL decay profiles $\left[I_{\sigma+}\right.$ (red curve) and $I_{\sigma}-$ (black curve)] and the time-resolved valley polarization, $\rho_{\mathrm{x}}(t)$, (green circles) measured at $45 \mathrm{~K}$ under the $\sigma_{+}$excitation condition. By extrapolating $\rho_{\mathrm{x}}(t)$ to the onset time $(t \approx 0 \mathrm{ps})$ of the PL decay signals (dotted line), the lower limit of the maximum valley polarization of $\rho_{0} \equiv \rho_{\mathrm{x}}(0) \approx 0.7 \pm 0.1$ is deduced. Since the similar value was also deduced at $120 \mathrm{~K}$ (see Supplementary Note 3 and Supplementary Figure 2 for additional details), temperature-dependent variation of the $\rho_{0}$ parameter is considered to be small in the observed temperature range, and will be neglected in the following analyses (See also Supplementary Note 4 and Supplementary Figure 3 for the effects of the uncertainty in the $\rho_{0}$ on the following analysis).

Temperature variations in exciton linewidth. Information about the temperature-dependent variations of the exciton homogeneous linewidths $\Gamma_{\mathrm{h}}$ were deduced from the PL spectral analysis. We evaluated $\Gamma_{\mathrm{h}}$ using a fitting procedure with Voigt functions (see Methods, Supplementary Note 5, and Supplementary Figure 4 for the detailed procedure). The inset in Fig. 2a shows an example of the peak deconvolution employed for the spectrum collected at $40 \mathrm{~K}$. The reasonable uncertainty range of $\Gamma_{\mathrm{h}}$ estimated from the fit analysis (gray-shaded region) is plotted as a function of temperature in Fig. 3c. The temperature dependence of $\Gamma_{\mathrm{h}}$ was phenomenologically modeled as $\Gamma_{\mathrm{h}}=\Gamma_{0}+\Gamma_{1} T+$ $\Gamma_{2}\left[\exp \left(E_{\mathrm{ph}} / k_{\mathrm{B}} T\right)-1\right]^{-1}$, where $k_{\mathrm{B}}$ is the Boltzmann constant, $\Gamma_{0}$ is the temperature-independent linewidth, $\Gamma_{1}$ and $\Gamma_{2}$ are the fitting parameters, and $E_{\mathrm{ph}}=31 \mathrm{meV}$ is assumed to be a typical optical phonon energy in $1 \mathrm{~L}-\mathrm{WSe}_{2}{ }^{61}$. For convenience, we fit the mean values of $\Gamma_{\mathrm{h}}$ within a reasonable range at each temperature and obtained a best fit with $\Gamma_{0}=7.3 \mathrm{meV}, \Gamma_{1}=0.004 \mathrm{meV} / \mathrm{K}$, and $\Gamma_{2}$ $=39 \mathrm{meV}$. The fitted curve is plotted in Fig. 3c. The observed temperature dependence is consistent with trends previously reported for $1 \mathrm{~L}-\mathrm{WSe}_{2}{ }^{60,62-64}$.
Valley relaxation time of the bright excitons. We now consider the relationship between the valley polarization $\rho_{\mathrm{x}}$ and the pure valley relaxation time $\tau_{\mathrm{v}}$ of the bright excitons. The timeintegrated valley polarization of the excitons can be expressed by the following phenomenological equation ${ }^{14,15,40,65}$ (see Supplementary Note 1 for additional details):

$$
\rho_{\mathrm{x}}=\frac{\rho_{0}}{1+\left\langle\tau_{\mathrm{x}}\right\rangle / \tau_{\mathrm{v}}} .
$$

We set $\rho_{0}=0.7$ for $1 \mathrm{~L}-\mathrm{WSe}_{2}$ in the following analyses according to the results discussed in the previous section. Parameter $\left\langle\tau_{\mathrm{x}}\right\rangle$ in Eq. (1) corresponds to an exciton population lifetime in the simplest case for which only the bright exciton levels are involved ${ }^{14}$. For the multi-exciton-level system we consider in this study (Fig. 1), $\left\langle\tau_{\mathrm{x}}\right\rangle$ is defined as an effective lifetime of the bright excitons that corresponds to the total (integrated) time in which a single exciton remains in its bright state before it recombines radiatively or nonradiatively (see also Supplementary Note 1). Equation (1) suggests that $\tau_{\mathrm{v}}$, which is defined as the pure valley relaxation time of the bright excitons, can be evaluated if $\rho_{0}$ and $\left\langle\tau_{\mathrm{x}}\right\rangle$ are given. This procedure enables the evaluation of the pure valley relaxation time of an exciton in its bright state, which can be directly compared against the theoretical results. Notably, Eq. (1) is valid under the condition that the valley relaxation of holes composing excitons is dominated by the intervalley scattering of the bright excitons regardless of the microscopic origin of the scattering.

We evaluated $\left\langle\tau_{\mathrm{x}}\right\rangle$ from the time-resolved PL decay profile of the bright excitons, $I(t)$, as $\left\langle\tau_{\mathrm{x}}\right\rangle=\int_{0}^{\infty} I(t) / I(0) \mathrm{d} t$, where $I(t)$ is obtained by a fitting of the experimental data using a model PL decay function. Figure $2 c$ shows representative PL decay profiles at $10,40,80$, and $160 \mathrm{~K}$ in semi-log plots (see also Supplementary Note 6 and Supplementary Figure 5 for the data in linear plots). The data at each temperature were fitted as a convolution of the instrumental response function (IRF) and a model double exponential function (light-green curves), $I(t)=C_{1} \exp \left(-t / \tau_{1}\right)+$ $C_{2} \exp \left(-t / \tau_{2}\right)$, and $\left\langle\tau_{\mathrm{x}}\right\rangle=\left(C_{1} \tau_{1}+C_{2} \tau_{2}\right) /\left(C_{1}+C_{2}\right)$ was obtained by the fitting procedure. Figure $3 \mathrm{~d}$ plots the obtained $\left\langle\tau_{\mathrm{x}}\right\rangle$ as a function of temperature (See Supplementary Note 7 and Supplementary Figure 6 for the consistency between the temperature dependences of the $\left\langle\tau_{\mathrm{x}}\right\rangle$ and the exciton PL intensity). The solid curves represent the fit obtained using a model for Eq. (5) described in the Methods section (and in Supplementary Note 1), where the exciton scattering pathways shown in Fig. $1 \mathrm{~b}$ were considered. We found that the temperature dependence of $\left\langle\tau_{\mathrm{x}}\right\rangle$ can be well fitted using Eq. (5) with the bright-dark energy splitting ${ }^{54-57} \Delta_{\text {bd }}$ in the range $30-47 \mathrm{meV}$, supporting the validity of the model of the multi-exciton-level system shown in Fig. 1 (see Supplementary Note 1 for more detailed discussion on the results of the fitting and limitation of the model).

Valley relaxation mechanism of excitons. We then deduced the $\tau_{\mathrm{v}}$ from the relation of Eq. (1), namely, $\tau_{\mathrm{v}}{ }^{-1}=\left\langle\tau_{\mathrm{x}}\right\rangle^{-1}\left(\rho_{0} / \rho_{\mathrm{x}}-1\right)$, using the experimentally deduced values of $\rho_{0}, \rho_{\mathrm{x}}$, and $\left\langle\tau_{\mathrm{x}}\right\rangle$ at each temperature. Figure $3 \mathrm{e}$ shows the obtained valley relaxation times at various temperatures (green circles). The $\tau_{\mathrm{v}}$ values under the low-temperature conditions $(<\sim 40 \mathrm{~K})$ were on the order of $10 \mathrm{ps}$ and exhibited plateau-like behavior. Here, we discuss the origin of the exciton valley relaxation in greater detail on the basis of the temperature dependence of the bright exciton valley pseudospin relaxation times, $\tau_{\mathrm{v}}$. According to previous theoretical predictions $^{32,39}$, we consider long-range $e-h$ exchange interactions (originally known as the Maialle-Silva-Sham (MSS) mechanism 
for exciton spin-depolarization in semiconductors ${ }^{66}$ ) screened by $2 \mathrm{D}$ electrons naturally doped in $1 \mathrm{~L}-\mathrm{WSe}_{2}$ to be the dominant relaxation mechanism of the valley pseudospin of the bright excitons ${ }^{32}$. In this theoretical framework, the inverse exciton valley relaxation time is given as, $\tau_{\mathrm{v}}{ }^{-1}=\left\langle\Omega_{\mathbf{k}}{ }^{2} \tau_{\mathrm{h}}\right\rangle$, where $\Omega_{\mathbf{k}}$ is the momentum-dependent Larmor frequency under an effective magnetic field and $\tau_{\mathrm{h}}$ is the momentum relaxation time, which is inversely proportional to $\Gamma_{\mathrm{h}}$ (See also Supplementary Note 8 ). Under low-temperature conditions $\left(\Gamma_{\mathrm{h}}>\sim k_{\mathrm{B}} T\right)$ with screening by doped carriers, $\tau_{\mathrm{v}}$ for bright excitons can be approximately expressed as ${ }^{32}$

$$
\frac{1}{\tau_{\mathrm{v}}}=\frac{A J^{2} \Gamma_{\mathrm{h}}}{\hbar} \frac{1}{k_{\mathrm{TF}}^{2}},
$$

where $A=9 a^{2} M^{2} / 4 \pi^{2} \hbar^{4}$. is a material-dependent constant in which $a$ is the lattice constant, $M$ is the exciton mass, $\hbar$ is Planck's constant divided by $2 \pi, J$ is the strength of the exchange interaction, and $k_{\mathrm{TF}}$ is the Thomas-Fermi wave vector; $k_{\mathrm{TF}}$ has a dimensionality-dependent function form and, for a $2 \mathrm{D}$ electron gas, $k_{\mathrm{TF}}\left(T, E_{\mathrm{F}}\right)=k_{\mathrm{TF} 0}\left[1-\exp \left(-E_{\mathrm{F}} / k_{\mathrm{B}} T\right)\right]^{32}$. Parameter $E_{\mathrm{F}}$ is the Fermi energy measured from the bottom of the conduction band, and it is related to the carrier density, $n_{\mathrm{c}}$, by $n_{\mathrm{c}}=g_{\mathrm{s}} g_{\mathrm{v}} m_{e} E_{\mathrm{F}} /\left(2 \pi \hbar^{2}\right)$, where $g_{\mathrm{s}}$ and $g_{\mathrm{v}}$ are the spin and valley degeneracies, respectively, and $m_{\mathrm{e}}$ is the electron mass. Parameter $k_{\mathrm{TF} 0}$, given by $k_{\mathrm{TF} 0}=g_{\mathrm{s}} g_{\mathrm{v}} m_{\mathrm{e}} e^{2} /\left(4 \pi \varepsilon \hbar^{2}\right)$, is a zero-temperature Thomas-Fermi wave vector. In the following analyses, $a=0.334 \mathrm{~nm}, m_{\mathrm{e}}=$ $0.38 m_{0}{ }^{67}$, and $M=2 m_{\mathrm{e}}$ were used for $1 \mathrm{~L}-\mathrm{WSe}_{2}$. Equation (2) is valid when $k_{\mathrm{h}} \ll k_{\mathrm{TF}}$, where $k_{\mathrm{h}}=\sqrt{2 M \Gamma_{\mathrm{h}} / \hbar^{2}}$ is a maximum wave vector of excitons determined by the collisional broadening; this condition is safely met for a carrier density of $n_{\mathrm{c}}>\sim 5 \times 10^{10} \mathrm{~cm}^{-2}$ for $\Gamma_{\mathrm{h}}<\sim 35 \mathrm{meV}$, which is normally fulfilled in naturally carrierdoped 1L-TMDCs.

The orange-shaded region in Fig. $3 e$ is the prediction band for valley relaxation times reproduced using Eq. (2) and the experimentally deduced values of $\Gamma_{h}$ within a reasonable uncertainty range (gray-shaded region in Fig. 3c) with fitting parameters of $E_{\mathrm{F}}\left(\propto n_{\mathrm{c}}\right)$ and $J$ for the screening function. The vertical width of the prediction band mainly originates from the uncertainty in $\Gamma_{\mathrm{h}}$. The temperature dependence of $\tau_{\mathrm{v}}$ deduced using Eq. (1) (solid circles) could be excellently reproduced using the theoretical model of Eq. (2) with the experimental $\Gamma_{\mathrm{h}}$, given the fitting parameters $E_{\mathrm{F}}=13 \mathrm{meV}\left(n_{\mathrm{c}} \approx 2.1 \times 10^{12} \mathrm{~cm}^{-2}\right)$ and $J$ $=0.83 \mathrm{eV}$. The implied $E_{\mathrm{F}}$ and the carrier density on the order of $10^{12} \mathrm{~cm}^{-2}$ is consistent with the observed trion PL intensity (see also Supplementary Note 9) and previous results for as-exfoliated $1 \mathrm{~L}-\mathrm{TMDCs}^{68}$. Within the $\mathbf{k} \cdot \mathbf{p}$ approximation, $J$ can be roughly estimated as $J \approx 8 \pi^{2} a E_{\mathrm{b}} t^{2} / 3 a_{\mathrm{B}} E_{\mathrm{g}}{ }^{2} 32,69$, where $E_{\mathrm{b}}$ is the excitonbinding energy, $t$ is the hopping energy, $E_{\mathrm{g}}$ is the band gap, and $a_{\mathrm{B}}$ is the exciton Bohr radius. Using $E_{\mathrm{b}}=0.37 \mathrm{eV}^{8}, t=1.19 \mathrm{eV}^{12}$, $E_{\mathrm{g}}=2.11 \mathrm{eV}$, and $1 \leq a_{\mathrm{B}} \leq 2 \mathrm{~nm}$ for $1 \mathrm{~L}-\mathrm{WSe}_{2}$, approximately $0.5 \leq J \leq 1 \mathrm{eV}$ is deduced. Thus, the fitting result of $J \approx 0.83 \mathrm{eV}$ is consistent with the theoretical prediction of the exchange interaction strength.

The lower-left inset of Fig. $3 \mathrm{e}$ shows a plot of the $E_{\mathrm{F}}$ dependence of $\tau_{\mathrm{v}}$ calculated using Eq. (2) and the empirical function for the mean values of $\Gamma_{h}$. The low-temperature values of $\tau_{\mathrm{v}}$ are clearly observed to be independent of $E_{\mathrm{F}}$, and the lowtemperature plateau is extended for larger $E_{\mathrm{F}}$ values (higher carrier density). For the intermediate temperatures $(\sim 40 \mathrm{~K}<T<$ $\sim 160 \mathrm{~K})$, the valley relaxation times will be longer when the carrier density is greater. For further high-temperature conditions, Eq. (2) may no longer be applicable. At high temperatures, valley depolarization mechanisms other than the screened $e-h$ exchange interactions, such as the intervalley scattering through the thermal activation of holes to the spin-degenerate $\Gamma$ valley ${ }^{22}$, may also coexist, which would lead to Arrhenius-type temperature dependence with an activation energy corresponding to the energy difference of the $\left|K_{\mathrm{e}} K_{\mathrm{h}}\right\rangle$ and $\left|K_{\mathrm{e}} \Gamma_{\mathrm{h}}\right\rangle$ excitons with electrons in the $\pm K$ valley and holes at the $\pm K$ or $\Gamma$ valley. The activation energy has been estimated to be on the order of $0.14 \mathrm{eV}$ for $1 \mathrm{~L}$ $\mathrm{WS}_{2}^{22}$; assuming a similar order for $1 \mathrm{~L}-\mathrm{WSe} e_{2}$, this scattering pathway becomes gradually important for $T>\sim 200 \mathrm{~K}$.

To show the manifestation of the $2 \mathrm{D}$ screening in $\tau_{\mathrm{v}}(T)$ more clearly, we plotted $\sqrt{A J^{2} \tau_{\mathrm{v}}(T) \Gamma_{\mathrm{h}}(T) / \hbar}$ in the right-top inset of Fig. $3 \mathrm{e}$, which corresponds to $k_{\mathrm{TF}}\left(T, E_{\mathrm{F}}\right)$ according to Eq. (2). The mean values of the estimated linewidth in the uncertainty range shown in Fig. $3 \mathrm{c}$ were used as $\Gamma_{\mathrm{h}}(T)$ at each temperature. The function form of the characteristic 2D Thomas-Fermi wave vector, $k_{\mathrm{TF}}\left(T, E_{\mathrm{F}}\right)$, excellently reproduces the temperature dependence originating from the term $\sqrt{\tau_{\mathrm{v}}(T) \Gamma_{\mathrm{h}}(T)}$. This plot and Eq. (2) also suggest that the dimensionless quantity $k_{\mathrm{TF} 0}^{2} / A J^{2}=\tau_{\mathrm{v}}(0) \Gamma_{\mathrm{h}}(0) / \hbar$ is a constant that is dependent only on the material and is directly accessible by experiment. From our current experimental results, we obtained $\tau_{\mathrm{v}}(0) \Gamma_{\mathrm{h}}(0) / \hbar \approx 118$ for $1 \mathrm{~L}-\mathrm{WSe}_{2}$. Although the aforementioned considerations suggest that the presented model could capture important physics in the exciton valley relaxation phenomenon in doped $1 \mathrm{~L}-\mathrm{WSe}_{2}$, nevertheless, we note that our theoretical treatment within the static approximation neglects the doping-dependent changes of the exciton-binding energy and the quasiparticle band gap ${ }^{68}$ dominated by the dynamical screening effect on the direct Coulomb interactions ${ }^{70}$, and any possible dynamical effect on the screening of the $e-h$ exchange interactions; these potential shortcomings may change the formula of the valley relaxation time both in a qualitative and quantitative manner (See Supplementary Note 10 for more detailed discussion). Thus, it is still desired to develop a more rigorous theoretical approach for in-depth understanding of the underlying physics on the valley relaxation phenomena in the carrier-doped $2 \mathrm{D}$ semiconductors.

Valley polarization engineering. On the basis of the aforementioned results, a semi-empirical formula for the temperature-, Fermi energy- (carrier density-), and linewidth-dependent $\tau_{\mathrm{v}}$ can be expressed as:

$$
\tau_{\mathrm{v}}\left(T, E_{\mathrm{F}}, \Gamma_{\mathrm{h}}\right)=\frac{C \hbar\left[1-\exp \left(-E_{\mathrm{F}} / k_{\mathrm{B}} T\right)\right]^{2}}{\Gamma_{\mathrm{h}}},
$$

where $C$ is a material-dependent dimensionless parameter $C \equiv$ $\left.k_{\mathrm{TF0}}{ }^{2} / \mathrm{A}\right)^{2}=\tau_{\mathrm{v}}(0) \Gamma_{\mathrm{h}}(0) / \hbar$ (see Supplementary Note 10 for further discussion on the material dependence of $C$ ). From the experimental values of $\tau_{\mathrm{v}}$ and $\Gamma_{\mathrm{h}}$ at the low-temperature condition, $C \approx$ 118 for the $1 \mathrm{~L}-\mathrm{WSe}_{2}$ was deduced in this study. Then, upon substitution of Eq. (3) into Eq. (1), the steady-state exciton valley polarization is predicted as:

$$
\rho_{\mathrm{x}}=\frac{\rho_{0}}{1+\left\langle\tau_{\mathrm{x}}\right\rangle \Gamma_{\mathrm{h}} / C \hbar\left[1-\exp \left(-E_{\mathrm{F}} / k_{\mathrm{B}} T\right)\right]^{2}} .
$$

Equation (4) suggests a condition in which the exciton valley polarization becomes high; the narrower linewidth $\Gamma_{\mathrm{h}}$, the larger $E_{\mathrm{F}}$ (carrier density), and the shorter $\left\langle\tau_{\mathrm{x}}\right\rangle$ will be the keys to achieving a longer $\tau_{\mathrm{v}}$ and a higher $\rho_{\mathrm{x}}$. We first examined the expected correlation between the $\rho_{\mathrm{x}}$ and the $\Gamma_{\mathrm{h}}$ by changing the excitation power density at $10 \mathrm{~K}$ using Eq. (4) at the lowtemperature limit $\rho_{\mathrm{x}} \approx \rho_{0}\left(1+\left\langle\tau_{\mathrm{x}}\right\rangle \Gamma_{\mathrm{h}} / C h\right)^{-1}$. The expected inverse correlation of $\rho_{\mathrm{x}}$ and $\Gamma_{\mathrm{h}}$ was actually observed and could be well reproduced using this simple relation, as shown in Supplementary 
Note 11 and Supplementary Figure 7. Moreover, we could also successfully apply the above relation for reproducing the excitation photon energy dependence of the valley polarization observed at $15 \mathrm{~K}$ from the $\left\langle\tau_{\mathrm{x}}\right\rangle$ and $\Gamma_{\mathrm{h}}$ observed at each excitation photon energy (Supplementary Note 2 and Supplementary Figure 1). The wide applicability of the aforementioned relation under low-temperature conditions thus strongly supports the validity of the presented mechanism.
To further verify Eq. (4) at the finite-temperature conditions and achieve realistic engineering on the excitonic valley polarization, we fabricated $1 \mathrm{~L}-\mathrm{WSe}_{2}$ stacked on a multilayer graphene flake, as shown in Fig. 4a (see Methods). For this type of sample, we expect that the exciton linewidth could be narrow because of the reduced scattering on the atomically flat surface of multilayer graphene $\mathrm{e}^{71}$ and because of the shorter $\left\langle\tau_{\mathrm{x}}\right\rangle$ resulting from charge or energy transfer to the graphene. Modulating the a

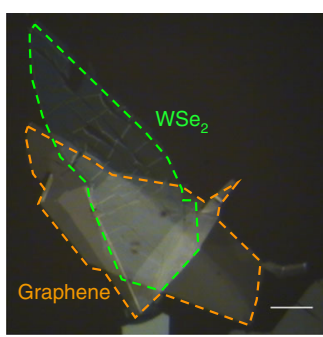

C

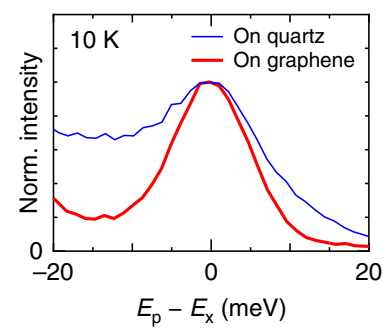

d

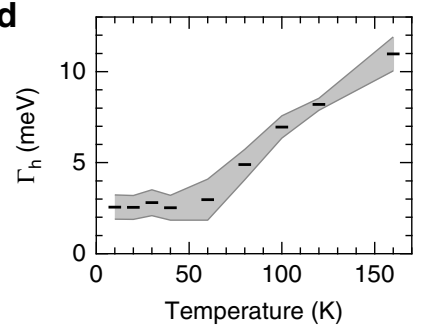

e

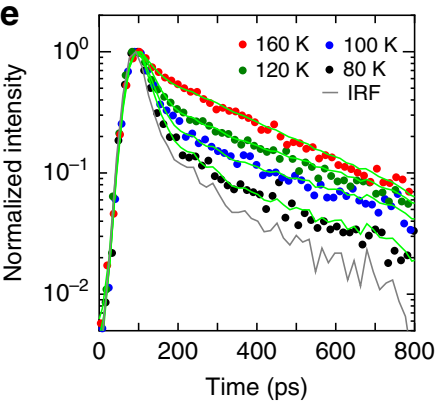

b
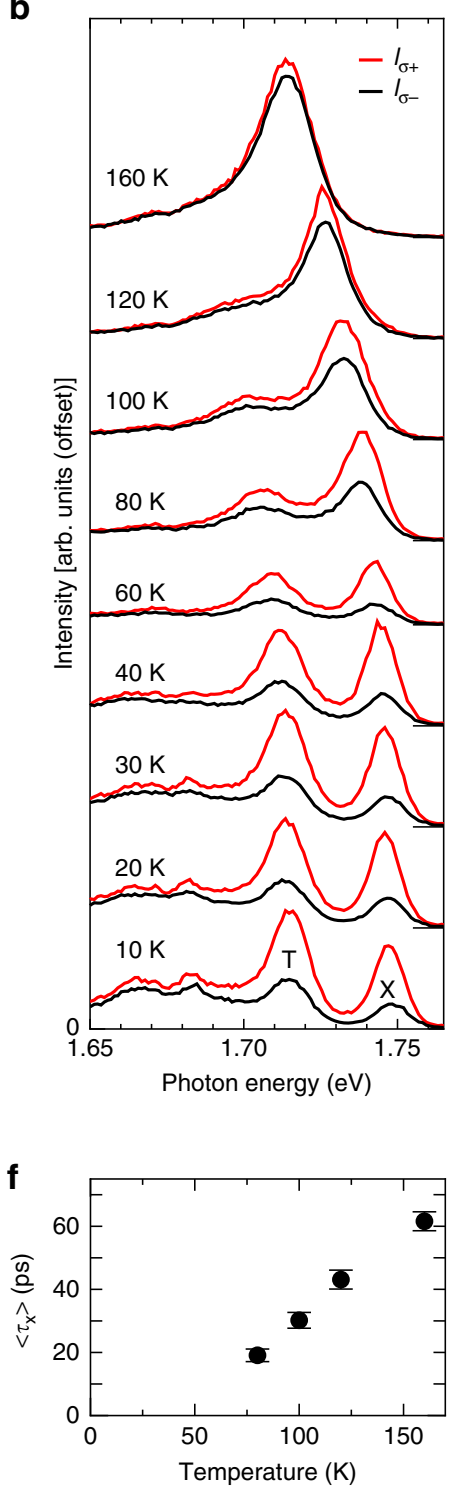

g

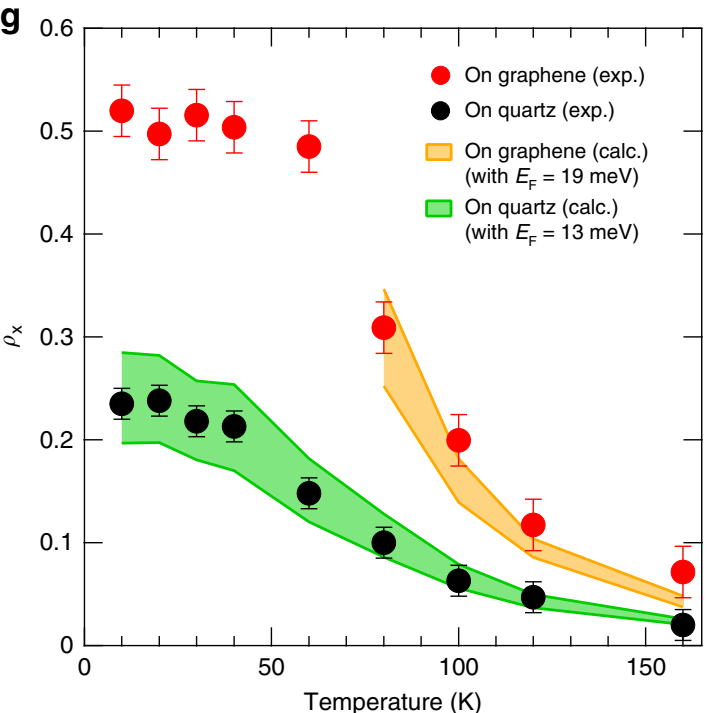

h 0.6

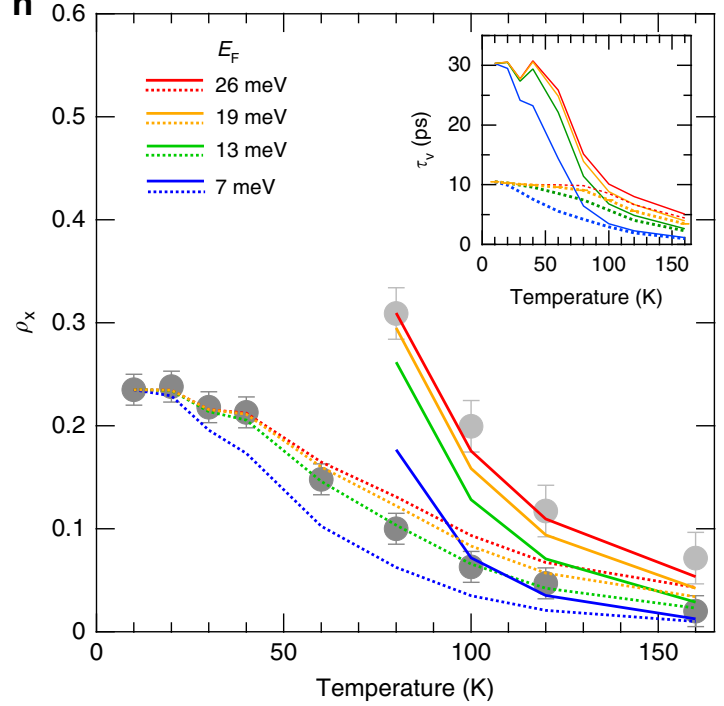

Fig. 4 Valley polarization engineering. a Optical microscope image of $1 \mathrm{~L}-\mathrm{WS} \mathrm{e}_{2}$ stacked on a multilayer graphene flake (on-graphene) on a quartz substrate. The scale bar indicates $10 \mu \mathrm{m}$. b Polarization-resolved PL spectra of the on-graphene $1 \mathrm{~L}-\mathrm{WSe} \mathrm{S}_{2}$ under $\sigma_{+}$excitation at 10-160 K. The red and black curves correspond to the $\sigma_{+}$and $\sigma-\mathrm{PL}$ intensities, $I_{\sigma+}$ and $l_{\sigma-}$, respectively. c Comparison of the exciton PL line shapes at $10 \mathrm{~K}$ for the on-graphene and the onquartz $1 \mathrm{~L}-\mathrm{WS} \mathrm{e}_{2}$ samples. $\mathbf{d} \Gamma_{\mathrm{h}}$ as a function of temperature for the on-graphene $1 \mathrm{~L}-\mathrm{WSe} \mathrm{e}_{2}$. Gray-shaded region indicates the uncertainty range of $\Gamma_{\mathrm{h}}$ deduced from the Voigt fitting procedure. Bars are the mean values in the uncertainty range at each temperature. e Time-resolved PL decay profiles of excitons in the on-graphene $1 \mathrm{~L}-\mathrm{WS} \mathrm{S}_{2}$ measured at $160 \mathrm{~K}$ (red), $120 \mathrm{~K}$ (green), $100 \mathrm{~K}$ (blue), and $80 \mathrm{~K}$ (black), respectively. $\mathbf{f}\left\langle\tau_{\times}\right\rangle$in the on-graphene $1 \mathrm{~L}$ $\mathrm{WSe}_{2}$ plotted as a function of temperature. The error bars represent the uncertainties in the fitting procedure. $\mathbf{g} \rho_{\mathrm{x}}$ as a function of temperature for the ongraphene $1 \mathrm{~L}-\mathrm{WSe} \mathrm{S}_{2}$ (red circles) compared with those of on-quartz (black circles, same data are shown in Fig. 3a) $1 \mathrm{~L}-\mathrm{WSe} \mathrm{e}_{2}$. The orange-shaded region is the prediction band of $\rho_{\mathrm{x}}$ for the on-graphene $1 \mathrm{~L}-\mathrm{WSe} \mathrm{S}_{2}$ calculated using Eq. (1); $\rho_{0}=0.7,\left\langle\tau_{\mathrm{x}}\right\rangle$ plotted in $\mathbf{f}$, and $\tau_{\mathrm{v}}$ calculated using Eq. (3) (with the $\Gamma_{\mathrm{h}}$ in the uncertainty range shown in $\mathbf{d}$ and $E_{\mathrm{F}}=19 \mathrm{meV}$ ) were used as input parameters for Eq. (1). The green-shaded region is a prediction band for the on-quartz $1 \mathrm{~L}-\mathrm{WSe}_{2}$ with $E_{\mathrm{F}}=13 \mathrm{meV}$. The error bars correspond to the uncertainties of the polarization-resolved measurements. $\mathbf{h} \rho_{\mathrm{x}}$ predicted for various $E_{\mathrm{F}}$ as functions of temperature. The inset shows $\tau_{\mathrm{v}}$ calculated using Eq. (3) with the mean values of $\Gamma_{\mathrm{h}}$ shown in $\mathbf{d}$ and various $E_{\mathrm{F}}$ values. Solid and dotted curves are for on-graphene and on-quartz samples, respectively 
carrier density in $1 \mathrm{~L}-\mathrm{WSe}_{2}$ may be possible because of the chemical potential difference between the graphene and $1 \mathrm{~L}-\mathrm{WSe}_{2}$.

Figure $4 \mathrm{~b}$ shows the temperature dependence of the exciton valley polarization in $1 \mathrm{~L}-\mathrm{WSe}_{2}$ stacked on the multilayer graphene substrate (on-graphene sample). For this sample, we observed a much higher exciton valley polarization $\rho_{\mathrm{x}}$ compared with those for the $1 \mathrm{~L}-\mathrm{WSe}_{2}$ on the quartz substrate (on-quartz sample), as compared in Fig. 4g. As expected, exciton linewidths much narrower than those on the quartz substrate were observed (Fig. 4c, d). In addition, the trion peak intensity was higher than the exciton's peak intensity, which suggests that the carrier density in this sample was higher than that in the sample on the quartz substrate. The trion/exciton intensity ratio for the ongraphene sample shown in Fig. $4 \mathrm{~b}$ is $\sim 1.5$ times greater than that for the on-quartz sample shown in Fig. 2. We also observed the PL decay profiles (Fig. 4e). We could obtain the $\left\langle\tau_{\mathrm{x}}\right\rangle$ for temperatures only more than $80 \mathrm{~K}$ as shown in Fig. $4 \mathrm{e}$, $\mathrm{f}$ because of the detection limit. The orange-shaded region in Fig. $4 \mathrm{~g}$ is the prediction band for the $\rho_{\mathrm{x}}$ reproduced using the $\left\langle\tau_{\mathrm{x}}\right\rangle$ and $\Gamma_{\mathrm{h}}$ values shown in Fig. 4f, d with $E_{\mathrm{F}}=19 \mathrm{meV}\left(n_{\mathrm{c}} \approx 3.0 \times 10^{12} \mathrm{~cm}^{-2}\right)$ as inputs to Eq. (4). For comparison, the prediction band for the onquartz sample (green-shaded region) calculated with the $\Gamma_{\mathrm{h}}$ shown in Fig. $3 \mathrm{c}$ and $E_{\mathrm{F}}=13 \mathrm{meV}\left(n_{\mathrm{c}} \approx 2.1 \times 10^{12} \mathrm{~cm}^{-2}\right)$ is also shown.

The valley polarization of the on-quartz sample and the increased valley polarization for the on-graphene sample could be consistently reproduced using Eq. (4). Importantly, the persistent valley polarization for $T>\sim 100 \mathrm{~K}$ in the on-graphene $1 \mathrm{~L}-\mathrm{WSe}_{2}$ cannot be explained by simply considering that the $\left\langle\tau_{\mathrm{x}}\right\rangle$ in the ongraphene sample is shorter than that in the on-quartz sample. For instance, at $100 \mathrm{~K}$, the $\rho_{\mathrm{x}}$ for the on-quartz sample was only $\sim 0.06$ and $\left\langle\tau_{\mathrm{x}}\right\rangle$ was $\sim 55 \mathrm{ps}$. The $\tau_{\mathrm{v}}$ was then deduced to be $\sim 5.5 \mathrm{ps}$, as shown in Fig. 3e. If $\left\langle\tau_{\mathrm{x}}\right\rangle$ becomes shorter, such as $\left\langle\tau_{\mathrm{x}}\right\rangle \approx 30 \mathrm{ps}$, as observed in the on-graphene sample, and the other parameters in Eq. (1) are kept unchanged, then $\rho_{\mathrm{x}}$ is expected to increase only slightly to $\sim 0.1$. This prediction contradicts the much higher $\rho_{\mathrm{x}} \approx$ 0.2 for the on-graphene $1 \mathrm{~L}-\mathrm{WSe}_{2}$ at $100 \mathrm{~K}$; thus, the narrower $\Gamma_{\mathrm{h}}$ and higher carrier density also contribute to the high-valley polarization, as predicted from Eq. (4). Figure 4 h shows the $E_{\mathrm{F}}$ dependence of the $\rho_{\mathrm{x}}$ predicted by Eq. (4). For clarity, we used the mean values of the $\Gamma_{\mathrm{h}}$ in the uncertainty ranges shown in Fig. $3 \mathrm{c}$ (on-quartz) and Fig. 4d (on-graphene). The high values of $\rho_{\mathrm{x}}$ for the on-graphene sample clearly cannot be reproduced with the same $E_{\mathrm{F}}$ (carrier density) as that for the on-quartz sample. The inset of Fig. $4 \mathrm{~h}$ shows the $\tau_{\mathrm{v}}$ for various $E_{\mathrm{F}}$ calculated using Eq. (3) and the mean values of the $\Gamma_{\mathrm{h}}$ for the corresponding samples. The plateau region of $\tau_{\mathrm{v}}$ is predicted to be extended as the $E_{\mathrm{F}}$ increased, consistent with the persistent $\rho_{\mathrm{x}}$ for the on-graphene sample at temperatures as high as $\sim 60 \mathrm{~K}$ (Fig. $4 \mathrm{~g}$ ). These results confirm that the presented framework provides clear guidelines for engineering exciton valley polarization in $1 \mathrm{~L}-\mathrm{WSe}_{2}$. We note that there remains a possibility that the $\rho_{0}$ and $C$ also vary depending on the carrier density and/or the substrate's dielectric constant. Thus, the prediction of Eq. (4) may be further modified when taking these effects into account (See Supplementary Note 10 for more detailed discussion).

\section{Discussion}

Finally, we discuss whether our results can be extended so as to predict the exciton valley relaxation times in $1 \mathrm{~L}-\mathrm{WSe}_{2}$ under high-excitation-density conditions employed in ultrafast spectroscopic studies using femtosecond lasers ${ }^{38,46}$. The temperature dependence of $\tau_{\mathrm{v}}$ deduced in this study, as shown in Fig. 3, is qualitatively similar to the temperature dependence of previous results obtained by TRKR measurements ${ }^{38,46}$; however, the absolute values we obtained are a few times greater than those previously reported. We address this discrepancy through the exciton density dependence of the linewidth. A previous study on the intrinsic excitonic linewidth in $1 \mathrm{~L}-\mathrm{WSe}_{2}{ }^{62}$ reported that $\Gamma_{\mathrm{h}}$ strongly depends on the excitation density, $N_{\mathrm{x}}$, as $\Gamma_{\mathrm{h}}=\Gamma_{\mathrm{s}}+$ $\Gamma_{\text {col }} N_{\mathrm{x}}$, under intense excitation conditions using femtosecond pulsed lasers, where $\Gamma_{\mathrm{s}}$ is a single exciton linewidth and $\Gamma_{\mathrm{col}} \approx$ $5.4 \times 10^{12} \mathrm{meV} \mathrm{cm}^{-2}$ is the constant for the density-dependent broadening due to exciton-exciton collisions ${ }^{62}$. Thus, we assume that the density- and temperature-dependent linewidths are expressed as $\Gamma_{\mathrm{h}}\left(T, N_{\mathrm{x}}\right)=\Gamma_{\mathrm{s}}(T)+\Gamma_{\text {col }} N_{\mathrm{x}}$, where $\Gamma_{\mathrm{s}}(T)$ is the linewidth observed under the weak excitation conditions and $\Gamma_{\mathrm{col}}$ is a temperature-independent proportionality constant.

Figure 5 shows the $\tau_{\mathrm{v}}$ for various exciton densities, $N_{\mathrm{x}}$, in $1 \mathrm{~L}$ $\mathrm{WSe}_{2}$ predicted using the exciton-density-dependent $\Gamma_{\mathrm{h}}\left(T, N_{\mathrm{x}}\right)$ as inputs for Eq. (3). For the temperature-dependent $\Gamma_{s}(T)$, we used the empirical formula obtained by fitting the mean values of $\Gamma_{\mathrm{h}}$ in the uncertainty range shown in Fig. $3 \mathrm{c}$ for all cases. Along with the experimental data obtained in this study (circles), the data reported in previous ultrafast TRKR studies on $1 \mathrm{~L}-\mathrm{WSe}_{2}{ }^{38,46}$ are also plotted as rectangles and triangles. As shown in Fig. 5, all of the data sets of $\tau_{\mathrm{v}}$ obtained in the experiments conducted under various excitation density conditions could be reproduced with appropriate $N_{\mathrm{x}}$ and $E_{\mathrm{F}}$ values as fitting parameters. The $N_{\mathrm{x}}$ values required to reproduce the data in ref. ${ }^{38}\left(1.0 \times 10^{12} \mathrm{~cm}^{-2}\right)$ and ref. $46\left(2.5 \times 10^{12} \mathrm{~cm}^{-2}\right)$ are in excellent agreement with the actual excitation densities used in these studies, which are on the orders of $\sim 10^{12} \mathrm{~cm}^{-238}$ and $\sim 2 \times 10^{12} \mathrm{~cm}^{-246}$. The ability to reproduce the experimental data for a wide range of excitation density conditions indicates the robustness and universality of the presented model even under high-exciton-density conditions. Our findings thus offer a unified framework to predict the exciton valley relaxation of $1 \mathrm{~L}-\mathrm{TMDC}$ observed under various experimental conditions. The results also provide clear guidelines for controlling the excitonic valley relaxation times via Fermi energy (carrier density) tuning and/or linewidth modulations via materials engineering and/or via optical means, which will facilitate

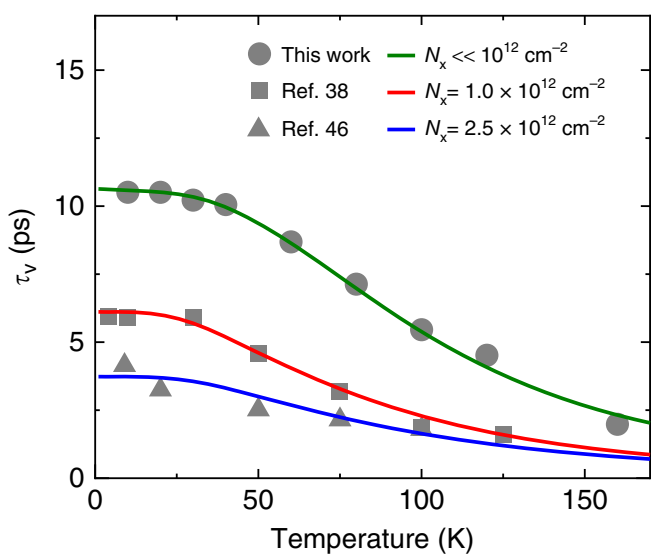

Fig. 5 Temperature dependence of exciton valley relaxation times predicted for various exciton density conditions. Temperature-dependent exciton valley relaxation times obtained for $1 \mathrm{~L}-\mathrm{WSe}_{2}$ on the quartz substrate in this study (circles), and those previously reported in ref. 38 (squares) and ref. 46 (triangles), both of which were measured using the TRKR technique. The green, red, and blue curves were reproduced using Eq. (3) and the densitydependent $\Gamma_{\mathrm{h}}\left(T, N_{\mathrm{x}}\right)$ with $N_{\mathrm{x}} \ll 10^{12} \mathrm{~cm}^{-2}\left(\Gamma_{\mathrm{h}}\left(0, N_{\mathrm{x}}\right)=7.3 \mathrm{meV}\right.$, green curve $), N_{\mathrm{x}}=1.0 \times 10^{12} \mathrm{~cm}^{-2}\left(\Gamma_{\mathrm{h}}\left(0, N_{\mathrm{x}}\right)=12.7 \mathrm{meV}\right)$, and $2.5 \times 10^{12} \mathrm{~cm}^{-2}$ $\left(\Gamma_{\mathrm{h}}\left(0, N_{\mathrm{x}}\right)=20.8 \mathrm{meV}\right.$, blue curve), respectively. The $E_{\mathrm{F}}$ parameters were set as $13 \mathrm{meV}\left(n_{\mathrm{c}} \approx 2.1 \times 10^{12} \mathrm{~cm}^{-2}\right)$ for the green curve, $9 \mathrm{meV}\left(n_{\mathrm{c}} \approx 1.4 \times\right.$ $\left.10^{12} \mathrm{~cm}^{-2}\right)$ for the red curve, and $10 \mathrm{meV}\left(n_{\mathrm{c}} \approx 1.6 \times 10^{12} \mathrm{~cm}^{-2}\right)$ for the blue curve, respectively, to reproduce the experimental temperature dependence 
the development of opto-valleytronic technologies based on $2 \mathrm{D}$ semiconductors.

\section{Methods}

Sample preparation. We used the mechanical exfoliation method to prepare the $1 \mathrm{~L}-\mathrm{WSe} \mathrm{e}_{2}$ samples on quartz substrates or on $300-\mathrm{nm}$-thick $\mathrm{SiO}_{2} / \mathrm{Si}$ substrates ${ }^{72}$. The $1 \mathrm{~L}-\mathrm{WSe}_{2}$ samples stacked on multilayer graphene flakes were fabricated through a dry-transfer method using dimethylpolysiloxane (PDMS) films ${ }^{73,74}$. The $1 \mathrm{~L}$-WSe $e_{2}$ and multilayer graphene flakes were mechanically exfoliated onto the PDMS films, and these flakes were stacked onto a quartz substrate under an optical microscope.

Optical measurements. For the circularly polarized excitation of $1 \mathrm{~L}-\mathrm{WSe}_{2}$, white light from a super-continuum light source was sent through a monochromator, a linear polarizing prism, a quarter-wave plate, and a 0.5 numerical aperture objective lens. The $\sigma_{+}\left(\sigma_{-}\right)$circularly polarized light was used for the initial excitation of the excitons with the valley pseudospin $|+K\rangle(|-K\rangle)$. The light emission from the sample was collected using the objective lens, and the $\sigma_{+}$and $\sigma_{-}$circularly polarized components were converted into two orthogonal linear polarized components using the quarter-wave plate. These linear polarized components were separated by a calcite polarizing beam displacer and sent to a spectrograph and detected on separated regions of a liquid-nitrogen-cooled charge-coupled device detector. A time-correlated single-photon counting method was used to perform the time-resolved PL spectroscopy under a pulsed excitation of duration of $20 \mathrm{ps}$ and a frequency of $40 \mathrm{MHz}$. In the time-resolved measurements, the emitted light was sent though band-pass filters (10-nm band width) that corresponded to the exciton resonance energies at each temperature and they were detected using a fiber-coupled single-photon avalanche photodiode device. Except for the results shown in Fig. 2b and Supplementary Fig. 2, light polarization was not resolved in the time-resolved PL measurements. For the temperature range below $\sim 40 \mathrm{~K}$, the fast components of the PL decays shown in Fig. $2 \mathrm{c}$ were approximately at the edge of the time resolution limit, whereas the slow components were more than a few hundreds of picoseconds. Thus, the values obtained for $\left\langle\tau_{\mathrm{x}}\right\rangle$ in the low-temperature range may be an upper limit. The excitation power density was $10^{2} \mathrm{~W} \mathrm{~cm}^{-2}$ for both the spectral and time-resolved measurements, except for the excitationpower-dependent measurements shown in Supplementary Fig. 7. We confirmed the linear dependence of the exciton PL intensity on the excitation density. The exciton linewidth exhibited no detectable broadening as the excitation power density increased in the vicinity of the conditions employed in this study $\left(\sim 10^{2} \mathrm{~W} \mathrm{~cm}{ }^{-2}\right)$ (except for the excitation-power-dependent observations using a power density $>\sim 4 \times 10^{2} \mathrm{~W} \mathrm{~cm}^{-2}$ shown in Supplementary Fig. 7).

Linewidth analysis. We evaluated the homogeneous linewidths, $\Gamma_{\mathrm{h}}$, of excitons using a fitting procedure with Voigt functions (convolutions of Lorentzian and Gaussian functions). The inset in Fig. 2a and Supplementary Figure 4 show the PL spectra that were decomposed by the Voigt fit at $40 \mathrm{~K}$ (Fig. 2a) or at four representative temperatures (Supplementary Fig. 4), respectively. The black circles are the experimental data and the green curves are the spectra reproduced by the peak fit. We considered the peak features for excitons (red curves) and the neighboring peaks of trions ${ }^{34}$ (gray curves) for the spectral decomposition; the major contributions of the neutral exciton $(\sim 1.740 \mathrm{eV})$ and negative trion $(\sim 1.706 \mathrm{eV})$, and one minor peak appearing at about $17 \mathrm{meV}$ below the exciton peak $(\sim 1.723 \mathrm{eV})$ were assumed. The minor PL feature at this energy range was also observed in the carrier-density-dependent PL spectra of $1 \mathrm{~L}-\mathrm{WSe}_{2}{ }^{34,59}$; the peak position implies a residual contribution from locally generated negative or positive trions with shifted energies at positions with relatively low carrier density, presumably because of spatial inhomogeneity of the carrier density on the $1 \mathrm{~L}-\mathrm{WSe}_{2}$ on a quartz substrate. Disappearance of this minor component for the high-carrier-doped sample on the atomically flat surface of the multilayer graphene shown in Fig. 4 supports this interpretation. Further details of the fitting procedure are described in Supplementary Note 5 .

Modeling for the temperature dependence of $\left\langle\tau_{\mathbf{x}}\right\rangle$. For convenience, in the discussion and validation of the model diagram shown in Fig. 1b, we considered a phenomenological formula to reproduce the experimental temperature dependence of $\left\langle\tau_{\mathrm{x}}\right\rangle$. Here, we model the temperature dependence of $\left\langle\tau_{\mathrm{x}}\right\rangle$ assuming a finite rate for phonon-mediated exciton scattering between the bright and dark states. We considered processes indicated by arrows in Fig. $1 \mathrm{~b}$ as the major exciton scattering pathways to express a non-equilibrium bright exciton distribution in a steady-state condition; the expression for $\left\langle\tau_{\mathrm{x}}\right\rangle$ is obtained as:

$$
\left\langle\tau_{\mathrm{x}}\right\rangle \equiv\left[\Gamma_{\mathrm{b}}+\frac{\Gamma_{\mathrm{d}} \exp \left(\Delta_{\mathrm{bd}} / k_{\mathrm{B}} T\right)}{1+\left(\Gamma_{\mathrm{d}} / \gamma\right)\left[\exp \left(\Delta_{\mathrm{bd}} / k_{\mathrm{B}} T\right)-1\right]}\right]^{-1},
$$

where $\Gamma_{b}$ and $\Gamma_{d}$ are the decay rates of the bright exciton and the dark exciton, respectively (see Supplementary Note 1 for the detailed derivation of this expression), and $\gamma$ is the rate constant for the intervalley phonon scattering. The solid curves in Fig. 3d are the fitted curves reproduced using Eq. (5). The fitted curve excellently reproduced the experimental temperature dependence of $\left\langle\tau_{\mathrm{x}}\right\rangle$ with an assumption of the previously reported bright-dark energy splitting $\Delta_{\mathrm{bd}}$ in the range from 30 to $47 \mathrm{meV}$ in $1 \mathrm{~L}-\mathrm{WSe}_{2}{ }^{54-57}$.

Data availability. The data that support the findings of this study are available from the corresponding authors on reasonable request.

Received: 14 May 2017 Accepted: 17 April 2018

Published online: 03 July 2018

\section{References}

1. Shkolnikov, Y. P., De Poortere, E. P., Tutuc, E. \& Shayegan, M. Valley splitting of AlAs two-dimensional electrons in a perpendicular magnetic field. Phys. Rev. Lett. 89, 226805 (2002).

2. Gunawan, O. et al. Valley susceptibility of an interacting two-dimensional electron system. Phys. Rev. Lett. 97, 186404 (2006).

3. Rycerz, A., Tworzydlo, J. \& Beenakker, C. W. J. Valley filter and valley valve in graphene. Nat. Phys. 3, 172-175 (2007).

4. Schaibley, J. R. et al. Valleytronics in 2D materials. Nat. Rev. Mater. 1, 16055 (2016).

5. Mak, K. F., Lee, C., Hone, J., Shan, J. \& Heinz, T. F. Atomically thin $\mathrm{MoS}_{2}$ : a new direct-gap semiconductor. Phys. Rev. Lett. 105, 136805 (2010).

6. Splendiani, A. et al. Emerging photoluminescence in monolayer $\mathrm{MoS}_{2}$. Nano Lett. 10, 1271-1275 (2010).

7. Ramasubramaniam, A. Large excitonic effects in monolayers of molybdenum and tungsten dichalcogenides. Phys. Rev. B 86, 115409 (2012).

8. He, K. et al. Tightly bound excitons in monolayer $\mathrm{WSe}_{2}$. Phys. Rev. Lett. 113, 026803 (2014).

9. Chernikov, A. et al. Exciton binding energy and nonhydrogenic Rydberg series in monolayer $\mathrm{WS}_{2}$. Phys. Rev. Lett. 113, 076802 (2014).

10. Mouri, S. et al. Nonlinear photoluminescence in atomically thin layered $\mathrm{WSe}_{2}$ arising from diffusion-assisted exciton-exciton annihilation. Phys. Rev. B 90, 155449 (2014).

11. Yu, H., Cui, X., Xu, X. \& Yao, W. Valley excitons in two-dimensional semiconductors. Natl. Sci. Rev. 2, 57-70 (2015).

12. Xiao, D., Liu, G.-B., Feng, W., Xu, X. \& Yao, W. Coupled spin and valley physics in monolayers of $\mathrm{MoS}_{2}$ and other group-VI dichalcogenides. Phys. Rev. Lett. 108, 196802 (2012)

13. Zeng, H., Dai, J., Yao, W., Xiao, D. \& Cui, X. Valley polarization in $\mathrm{MoS}_{2}$ monolayers by optical pumping. Nat. Nanotechnol. 7, 490-493 (2012).

14. Mak, K. F., He, K., Shan, J. \& Heinz, T. F. Control of valley polarization in monolayer $\mathrm{MoS}_{2}$ by optical helicity. Nat. Nanotechnol. 7, 494-498 (2012).

15. Cao, T. et al. Valley-selective circular dichroism of monolayer molybdenum disulphide. Nat. Commun. 3, 887 (2012).

16. Tatsumi, Y., Ghalamkari, K. \& Saito, R. Laser energy dependence of valley polarization in transition metal dichalcogenides. Phys. Rev. B 94, 235408 (2016).

17. Kioseoglou, G. et al. Valley polarization and intervalley scattering in monolayer $\mathrm{MoS}_{2}$. Appl. Phys. Lett. 101, 221907 (2012).

18. Sallen, G. et al. Robust optical emission polarization in $\mathrm{MoS}_{2}$ monolayers through selective valley excitation. Phys. Rev. B 86, 081301 (2012).

19. Mai, C. et al. Many-body effects in valleytronics: direct measurement of valley lifetimes in single-layer $\mathrm{MoS}_{2}$. Nano Lett. 14, 202-206 (2013).

20. Glazov, M. M. et al. Exciton fine structure and spin decoherence in monolayers of transition metal dichalcogenides. Phys. Rev. B 89, 201302 (2014).

21. Jones, A. M. et al. Spin-layer locking effects in optical orientation of exciton spin in bilayer WSe $\mathrm{W}_{2}$. Nat. Phys. 10, 130-134 (2014).

22. Mai, C. et al. Exciton valley relaxation in a single layer of $\mathrm{WS}_{2}$ measured by ultrafast spectroscopy. Phys. Rev. B 90, 041414 (2014).

23. Xu, X., Yao, W., Xiao, D. \& Heinz, T. F. Spin and pseudospins in layered transition metal dichalcogenides. Nat. Phys. 10, 343-350 (2014).

24. Mak, K. F., McGill, K. L., Park, J. \& McEuen, P. L. The valley hall effect in $\mathrm{MoS}_{2}$ transistors. Science 344, 1489-1492 (2014).

25. Kim, J. et al. Ultrafast generation of pseudo-magnetic field for valley excitons in $\mathrm{WSe}_{2}$ monolayers. Science 346, 1205-1208 (2014)

26. Konabe, S. \& Yamamoto, T. Valley photothermoelectric effects in transitionmetal dichalcogenides. Phys. Rev. B 90, 075430 (2014).

27. Zhang, Y. J., Oka, T., Suzuki, R., Ye, J. T. \& Iwasa, Y. Electrically switchable chiral light-emitting transistor. Science 344, 725-728 (2014).

28. Yan, T., Qiao, X., Tan, P. \& Zhang, X. Valley depolarization in monolayer WSe 2 . Sci. Rep. 5, 15625 (2015).

29. Srivastava, A. et al. Valley zeeman effect in elementary optical excitations of monolayer WSe $e_{2}$. Nat. Phys. 11, 141-147 (2015). 
30. Aivazian, G. et al. Magnetic control of valley pseudospin in monolayer $\mathrm{WSe}_{2}$. Nat. Phys. 11, 148-152 (2015).

31. Huang, J., Hoang, T. B. \& Mikkelsen, M. H. Probing the origin of excitonic states in monolayer $\mathrm{WSe}_{2}$. Sci. Rep. 6, 22414 (2016).

32. Konabe, S. Screening effects due to carrier doping on valley relaxation in transition metal dichalcogenide monolayers. Appl. Phys. Lett. 109, 073104 (2016).

33. Rivera, P. et al. Valley-polarized exciton dynamics in a $2 \mathrm{D}$ semiconductor heterostructure. Science 351, 688-691 (2016).

34. Jones, A. M. et al. Optical generation of excitonic valley coherence in monolayer $\mathrm{WSe}_{2}$. Nat. Nanotechnol. 8, 634-638 (2013).

35. Wang, Q. et al. Valley carrier dynamics in monolayer molybdenum disulfide from helicity-resolved ultrafast pump-probe spectroscopy. ACS Nano 7, 11087-11093 (2013).

36. Lu, H.-Z., Yao, W., Xiao, D. \& Shen, S.-Q. Intervalley scattering and localization behaviors of spin-valley coupled dirac fermions. Phys. Rev. Lett. 110, 016806 (2013)

37. Song, Y. \& Dery, H. Transport theory of monolayer transition-metal dichalcogenides through symmetry. Phys. Rev. Lett. 111, 026601 (2013).

38. Zhu, C. R. et al. Exciton valley dynamics probed by Kerr rotation in $\mathrm{WSe}_{2}$ monolayers. Phys. Rev. B 90, 161302 (2014).

39. $\mathrm{Yu}, \mathrm{T}$. \& Wu, M. W. Valley depolarization due to intervalley and intravalley electron-hole exchange interactions in monolayer $\mathrm{MoS}_{2}$. Phys. Rev. B 89, 205303 (2014)

40. Lagarde, D. et al. Carrier and polarization dynamics in monolayer $\mathrm{MoS}_{2}$. Phys. Rev. Lett. 112, 047401 (2014).

41. Wang, G. et al. Valley dynamics probed through charged and neutral exciton emission in monolayer $\mathrm{WSe}_{2}$. Phys. Rev. B 90, 075413 (2014).

42. Dery, H. \& Song, Y. Polarization analysis of excitons in monolayer and bilayer transition-metal dichalcogenides. Phys. Rev. B 92, 125431 (2015).

43. Dal Conte, S. et al. Ultrafast valley relaxation dynamics in monolayer $\mathrm{MoS}_{2}$ probed by nonequilibrium optical techniques. Phys. Rev. B 92, 235425 (2015).

44. Yang, L. et al. Long-lived nanosecond spin relaxation and spin coherence of electrons in monolayer $\mathrm{MoS}_{2}$ and $\mathrm{WS}_{2}$. Nat. Phys. 11, 830-834 (2015).

45. Schmidt, R. et al. Ultrafast coulomb-induced intervalley coupling in atomically thin $\mathrm{WS}_{2}$. Nano Lett. 16, 2945-2950 (2016).

46. Yan, T., Ye, J., Qiao, X., Tan, P. \& Zhang, X. Exciton valley dynamics in monolayer $\mathrm{WSe}_{2}$ probed by the two-color ultrafast Kerr rotation. Phys. Chem. Chem. Phys. 19, 3176-3181 (2017).

47. Huang, J., Hoang, T. B., Ming, T., Kong, J. \& Mikkelsen, M. H. Temporal and spatial valley dynamics in two-dimensional semiconductors probed via Kerr rotation. Phys. Rev. B 95, 075428 (2017).

48. Hanbicki, A. T. et al. Anomalous temperature-dependent spin-valley polarization in monolayer $\mathrm{WS}_{2}$. Sci. Rep. 6, 18885 (2016).

49. Plechinger, G. et al. Trion fine structure and coupled spin-valley dynamics in monolayer tungsten disulfide. Nat. Commun. 7, 12715 (2016).

50. Song, X., Xie, S., Kang, K., Park, J. \& Sih, V. Long-lived hole spin/valley polarization probed by Kerr rotation in monolayer $\mathrm{WSe}_{2}$. Nano Lett. 16, 5010-5014 (2016).

51. Yang, L. et al. Spin coherence and dephasing of localized electrons in monolayer $\mathrm{MoS}_{2}$. Nano Lett. 15, 8250-8254 (2015).

52. Hsu, W.-T. et al. Optically initialized robust valley-polarized holes in monolayer $\mathrm{WSe}_{2}$. Nat. Commun. 6, 8963 (2015).

53. Kozawa, D. et al. Photocarrier relaxation pathway in two-dimensiona semiconducting transition metal dichalcogenides. Nat. Commun. 5, 4543 (2014).

54. Zhang, X.-X., You, Y., Zhao, S. Y. F. \& Heinz, T. F. Experimental evidence for dark excitons in monolayer $\mathrm{WSe}_{2}$. Phys. Rev. Lett. 115, 257403 (2015).

55. Zhang, X.-X. et al. Magnetic brightening and control of dark excitons in monolayer $\mathrm{WSe}_{2}$. Nat. Nanotechnol. 12, 883-888 (2017).

56. Zhou, Y. et al. Probing dark excitons in atomically thin semiconductors via near-field coupling to surface plasmon polaritons. Nat. Nanotechnol. 12, 856-860 (2017).

57. Wang, G. et al. In-plane propagation of light in transition metal dichalcogenide monolayers: Optical selection rules. Phys. Rev. Lett. 119, 047401 (2017)

58. Zhao, W. et al. Evolution of electronic structure in atomically thin sheets of $\mathrm{WS}_{2}$ and $\mathrm{WSe}_{2}$. ACS Nano 7, 791-797 (2013).

59. Wang, Z., Shan, J. \& Mak, K. F. Valley- and spin-polarized landau levels in monolayer $\mathrm{WSe}_{2}$. Nat. Nanotechnol. 12, 144-149 (2016).

60. Arora, A. et al. Excitonic resonances in thin films of $\mathrm{WSe}_{2}$ : from monolayer to bulk material. Nanoscale 7, 10421-10429 (2015).

61. del Corro, E. et al. Excited excitonic states in 1l, 2l, 31, and bulk WSe $\mathrm{H}_{2}$ observed by resonant Raman spectroscopy. ACS Nano 8, 9629-9635 (2014).

62. Moody, G. et al. Intrinsic homogeneous linewidth and broadening mechanisms of excitons in monolayer transition metal dichalcogenides. Nat. Commun. 6, 8315 (2015)
63. Dey, P. et al. Optical coherence in atomic-monolayer transition-metal dichalcogenides limited by electron-phonon interactions. Phys. Rev. Lett. 116, 127402 (2016).

64. Selig, M. et al. Excitonic linewidth and coherence lifetime in monolayer transition metal dichalcogenides. Nat. Commun. 7, 13279 (2016).

65. Neumann, A. et al. Opto-valleytronic imaging of atomically thin semiconductors. Nat. Nanotechnol. 12, 329-334 (2017).

66. Maialle, M. Z., de Andrada e Silva, E. A. \& Sham, L. J. Exciton spin dynamics in quantum wells. Phys. Rev. B 47, 15776-15788 (1993).

67. Amin, B., Kaloni, T. P. \& Schwingenschlogl, U. Strain engineering of $\mathrm{WS}_{2}$, $\mathrm{WSe}_{2}$, and $\mathrm{WTe}_{2}$. RSC Adv. 4, 34561-34565 (2014).

68. Chernikov, A. et al. Electrical tuning of exciton binding energies in monolayer $\mathrm{WS}_{2}$. Phys. Rev. Lett. 115, 126802 (2015).

69. Yu, H., Liu, G.-B., Gong, P., Xu, X. \& Yao, W. Dirac cones and dirac saddle points of bright excitons in monolayer transition metal dichalcogenides. Nat. Commun. 5, 3876 (2014).

70. Gao, S., Liang, Y., Spataru, C. D. \& Yang, L. Dynamical excitonic effects in doped two-dimensional semiconductors. Nano Lett. 16, 5568-5573 (2016).

71. Kobayashi, Y. et al. Growth and optical properties of high-quality monolayer $\mathrm{WS}_{2}$ on graphite. ACS Nano 9, 4056-4063 (2015).

72. Novoselov, K. S. et al. Two-dimensional atomic crystals. Proc. Natl Acad. Sci. USA 102, 10451-10453 (2005).

73. Meitl, M. A. et al. Transfer printing by kinetic control of adhesion to an elastomeric stamp. Nat. Mater. 5, 33-38 (2006).

74. Mouri, S. et al. Thermal dissociation of inter-layer excitons in $\mathrm{MoS}_{2} / \mathrm{MoSe}_{2}$ hetero-bilayers. Nanoscale 9, 6674-6679 (2017).

\section{Acknowledgements}

This research was supported by JSPS KAKENHI Grant Numbers JP16H00911, JP15K13337, JP15H05408, JP15F15313, JP26390007, JP26107522, JP25400324, JP26390007, JP15K13500, JP16H00910, JP16H06331, and JP17K19055, by the Asahi Glass Foundation, IST Nanotech CUPAL, JST CREST (JPMJCR16F3), the Murata Science Foundation, the Research Foundation for Opto-Science and Technology, and the Nakatani Foundation. G.E. acknowledges Singapore National Research Foundation, Prime Minister's Office, Singapore for funding the research under NRF Research Fellowship (NRF-NRFF2011-02) and its Medium Sized Centre Program. G.E. also acknowledges support from the Ministry of Education (MOE), Singapore, under AcRF Tier 2 (MOE2015-T2-2-123, MOE2017-T2-1-134). The authors thank Y. Miyata and K. Shinokita for fruitful discussions.

\section{Author contributions}

Y.M. conceived the research and prepared the experimental set-up. Y.M. and S.K. analyzed and interpreted the data and wrote the manuscript. F.W., W.Z., A.H., Y.H., and L.Z. prepared the samples, performed the optical measurements, and contributed to analyzing of the data. M.T. and G.E. provided the samples. Y.M., S.K., S.M., and K.M. contributed to the interpretations of the results and writing the manuscript.

\section{Additional information}

Supplementary Information accompanies this paper at https://doi.org/10.1038/s41467018-04988-x.

Competing interests: The authors declare no competing interests.

Reprints and permission information is available online at http://npg.nature.com/ reprintsandpermissions/

Publisher's note: Springer Nature remains neutral with regard to jurisdictional claims in published maps and institutional affiliations.

\footnotetext{
Open Access This article is licensed under a Creative Commons Attribution 4.0 International License, which permits use, sharing, adaptation, distribution and reproduction in any medium or format, as long as you give appropriate credit to the original author(s) and the source, provide a link to the Creative Commons license, and indicate if changes were made. The images or other third party material in this article are included in the article's Creative Commons license, unless indicated otherwise in a credit line to the material. If material is not included in the article's Creative Commons license and your intended use is not permitted by statutory regulation or exceeds the permitted use, you will need to obtain permission directly from the copyright holder. To view a copy of this license, visit http://creativecommons.org/ licenses/by/4.0/.
}

(C) The Author(s) 2018 